Cytologia Focus:

\title{
Chromatin Live Imaging with Genome Editing Techniques: Switching from Scissors to a Lamp
}

\author{
Satoru Fujimoto and Sachihiro Matsunaga* \\ Department of Applied Biological Science, Faculty of Science and Technology, Tokyo University of Science, \\ 2641 Yamazaki, Noda, Chiba 278-8510, Japan \\ Received October 24, 2016; accepted November 1, 2016
}

\begin{abstract}
Summary Chromatin live imaging integrated with programmable DNA binding proteins derived from genome editing methods opens up the era of spatio-temporal analyses of chromatin dynamics. The DNA binding proteins, including a transcription activator-like effector (TALE) or a nuclease-dead CRISPR-associated protein 9 (dCas9), can be freely designed to bind a specific DNA sequence. Instead of a nuclease for DNA cleavage, a fluorescent protein is fused to TALE or dCAS9 for visualization of chromatin. They enable us to observe the endogenous genomic loci without the fixation of cells, the denaturation of DNA for hybridization and the insertion of exogenous DNA sequences into targeted loci. Using these live imaging systems dependent on the interaction between cis endogenous DNA sequence and trans DNA binding proteins, we can analyze chromatin dynamics in living cells. Multicolor chromatin imaging based on different dCas9 proteins will be a powerful cytogenetical technique instead of multicolor FISH and chromosome painting.
\end{abstract}

Key words Genome editing, TALE, dCAS9, Chromatin dynamics, Live cell imaging.

Chromatin dynamics plays an important role in the regulation of gene expression during development and differentiation (Schneider and Grosschedl 2007, Sexton et al. 2007, Rosa et al. 2013, Pontvianne et al. 2013, Matsunaga et al. 2013, Feng et al. 2014). Fluorescence in situ hybridization (FISH) has been useful to detect DNA distribution in nuclei and on chromosomes (Hoshi et al. 2011, Shibata and Hizume 2011, Ebadi-Almas et al. 2012, Shirakawa et al. 2012, Suto et al. 2012, Hizume et al. 2013, Ikeda et al. 2013, Kuroki et al. 2013, Ruan et al. 2013, Yokomi et al. 2013, Suto et al. 2013, Lombello and Pinto-Maglio 2014, Abo-Zeid et al. 2015, Kantek et al. 2015, Shibata and Hizume 2015, Matsunaga 2016). However, FISH supplies only a snapshot of subnuclear dynamics because it requires the fixation of cells and DNA denaturation with high temperature for hybridization. To analyze chromatin dynamics, live imaging methods were developed for visualization of chromatins (Bystricky 2015). Various chromatin proteins including histones, condensins and a proliferating cell nuclear antigen can be fused with fluorescent proteins for chromatin visualization (Kanda et al. 1998, Kimura and Cook 2001, Boisnard-Lorig et al. 2001, Gerlich et al. 2003, Fang and Spector 2005, Fujimoto et al. 2005, Lermontova et al. 2006, Higashi et al. 2007, Takata et al. 2007, Matsunaga and Umeda 2011, Nozaki

\footnotetext{
* Corresponding author, e-mail: sachi@rs.tus.ac.jp

DOI: $10.1508 /$ cytologia. 81.359
}

et al. 2014, Yokoyama et al. 2016). However, these protein-based chromatin visualization methods label whole or partial chromatins and are not available for the detection of specific genomic loci. The next approach was based on fluorescent repressor/operator systems, such as the lacO/LacI system (Robinett et al. 1996, Hirakawa et al. 2015, Hirakawa and Matsunaga 2016a). The fluorescent bacterial repressor fusion protein binds to the operator sequence inserted in the genome (Hirakawa and Matsunaga 2016b). Although twin dots on the homologous loci can be detected by fluorescent microscopy, the insertion of $\sim 9 \mathrm{~kb}$ of artificial tandem operator repeats into the host genome sometimes induces the accumulation of DNA methylation in the inserted site and influences chromatin dynamics (Jovtchev et al. 2011).

To reinforce the weakness in conventional methods, live imaging methods based on programmable DNA binding proteins derived from genome editing techniques were developed (Fig. 1). These methods do not require the insertion of exogenous DNA sequences but depend on the interaction between cis elements (endogenous DNA sequences) and trans regulatory proteins (DNA binding proteins). A first-generation programmable DNA binding protein, a zinc finger protein fused to GFP, was used for the visualization of repetitive DNA sequences (Lindhout et al. 2007). However, the zinc finger protein is limited in its target site selection because of context-dependent interactions with neighboring zinc fingers (Gaj et al. 2013). 

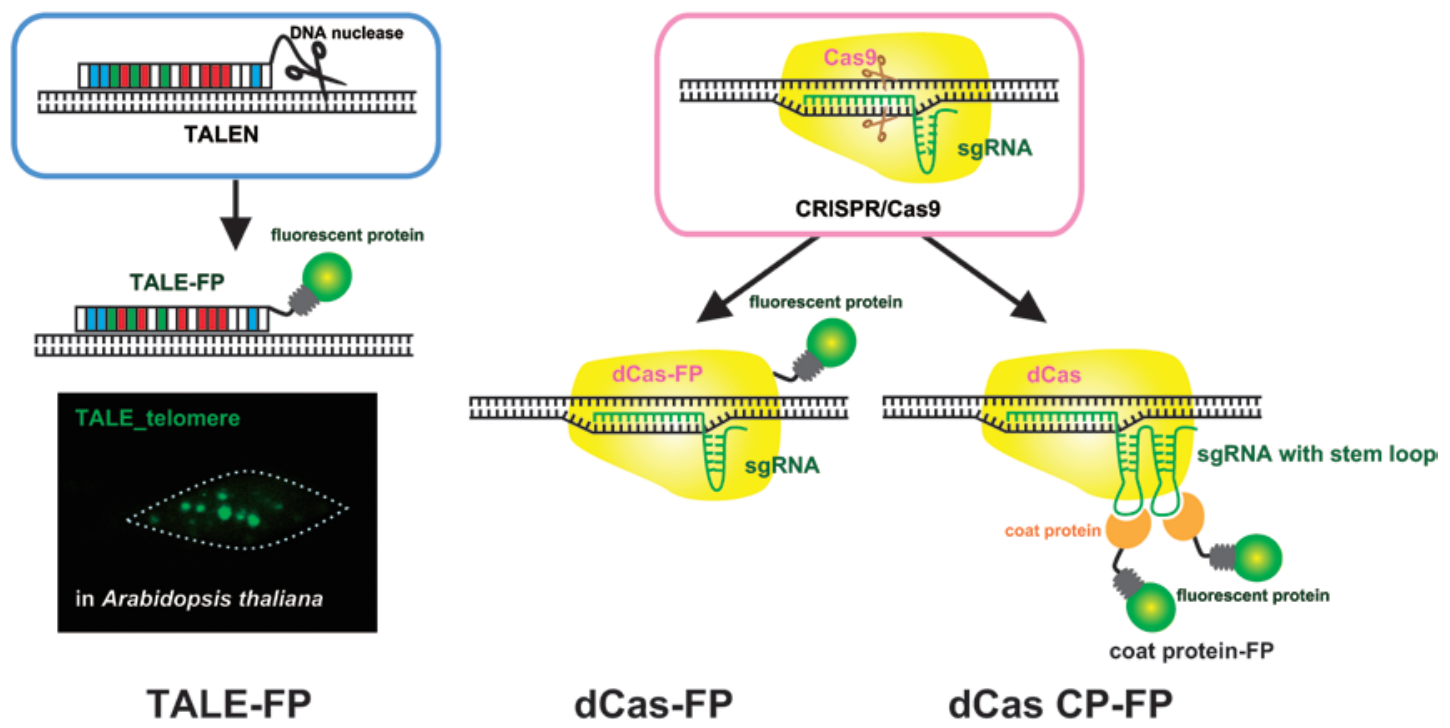

TALE-FP

dCas-FP

dCas CP-FP

Fig. 1. Chromatin live imaging with programmable DNA binding proteins, TALE-FP, dCas-FP and dCas CP-FP.

To overcome the limitations and complexity in design of DNA binding specificity, the codes of nucleotide recognized by repeat variable diresidues (RVDs) have been established in a second-generation programmable DNA binding protein, a transcription activator-like effector (TALE)-binding site derived from plant pathogenic bacteria Xanthomonas spp. (Boch et al. 2009, Moscou and Bogdanove 2009). By combining an engineered TALE with fluorescent proteins (TALE-FP) (also known as TGV, TALE-color, TALE-light and dTALE), TALEs are used for the visualization of repetitive sequences including centromeres and telomeres in mammalian cultured cells (Miyanari et al. 2013, Ma et al. 2013, Thanisch et al. 2014, Miyanari 2016). This system was also used for the visualization of 359-bp repetitive sequences on the $\mathrm{X}$ chromosome and $10 \mathrm{bp}$-repetitive sequences in the pericentomeric region in Drosophila melanogaster (Yuan et al. 2014, Yuan and O'Farrell 2016). This system was also successful in the visualization of telomeric sequences, 180-bp centromeric sequences, and 18S and 5S rDNA sequences in Arabidopsis thaliana (Fig. 1) (Fujimoto et al. 2016).

Systems of a third generation programmable DNA binding protein, clustered regularly interspaced short palindromic repeats (CRISPR)/CRISPR-associated protein 9 (Cas9), are also used for genome visualization (Chen et al. 2016a). A CRISPR/Cas9 system is a two-component system consisting of Cas9 from Streptococcus pyogenes and a single guide RNA (sgRNA), which directly interacts with the Cas9 nuclease at a specific locus in the genome (Jinek et al. 2012). Nuclease-dead Cas9 (dCas9) was fused with fluorescent proteins (dCas-FP) and cointroduced with sgRNAs for the target site (Fig. 1) (Chen et al. 2013, Anton et al. 2014, Lane et al. 2015). When multiple sgRNAs were introduced to the cells, even nonrepetitive single locus could be visualized (Chen et al. 2013). Multicolor versions of dCas-FP were developed using dCas from other bacterial orthologues (Ma et al. 2015, Chen et al. 2016b). Furthermore, to enable simultaneous visualization of multiple genomic regions with higher sensitivity, three-component systems were developed using dCas, sgRNA with stem loop motifs and the stem loop RNA binding coat proteins with fluorescent proteins (Fig. 1) (dCas CP-FP) (Ma et al. 2016, Shao et al. 2016, Wang et al. 2016, Fu et al. 2016). In this system, only one dCas is needed, and each fluorescent signal can be separated by the RNA stem loop motif-the coat protein pair. Moreover, a repeating peptide array, SunTag, which binds a single-chain fragment variable fused to GFP, was developed for signal amplification (Tanenbaum et al. 2014). Multiplex imaging with signal amplification will improve the ratio of signal to noise and enable us to analyze reliable chromatin dynamics on a single locus.

The relationship between chromatin mobility and double strand breaks (Lottersberger et al. 2015) or gene expression (Ochiai et al. 2015) was analyzed by these chromatin live imaging techniques. In the near future, chromatin live imaging with multicolor dCas-FP and multiple guide RNAs will allow simultaneous visualization of multiple specific chromosomes instead of multicolor FISH and chromosome painting. The advance in chromatin live imaging will contribute to produce new findings in DNA recombination, chromosome rearrangement, epigenetic regulation and nucleus organization.

\section{Acknowledgements}

This work was supported by CREST grants from the Japan Science and Technology Agency to S. M. and MEXT/JSPS KAKENHI (JP25120726, JP26291067 and JP15H05962) to S. M. 


\section{References}

Abo-Zeid, M. A. M., Liehr, T., Glei, M., Gamal-Eldeen, A. M., Zawrah, M. and Ali, M. 2015. Detection of cyto- and genotoxicity of rodshaped gold nanoparticles in human blood lymphocytes using comet-FISH. Cytologia 80: 173-181.

Anton, T., Bultmann, S., Leonhardt, H. and Markaki, Y. 2014. Visualization of specific DNA sequences in living mouse embryonic stem cells with a programmable fluorescent CRISPR/Cas system. Nucleus 5: 163-172.

Boch, J., Scholze, H., Schornack, S., Landgraf, A., Hahn, S., Kay, S., Lahaye, T., Nickstadt, A. and Bonas, U. 2009. Breaking the code of DNA binding specificity of TAL-type III effectors. Science 326: $1509-1512$.

Boisnard-Lorig, C., Colon-Carmona, A., Bauch, M., Hodge, S., Doerner, P., Bancharel, E., Dumas, C., Haseloff, J. and Berger, F. 2001. Dynamic analyses of the expression of the HISTONE:YFP fusion protein in Arabidopsis show that syncytial endosperm is divided in mitotic domains. Plant Cell 13: 495-509.

Bystricky, K. 2015. Chromosome dynamics and folding in eukaryotes: Insights from live cell microscopy. FEBS Lett. 589: 3014-3022.

Chen, B., Gilbert, L. A., Cimini, B. A., Schnitzbauer, J., Zhang, W., Li, G. W., Park, J., Blackburn, E. H., Weissman, J. S., Qi, L. S. and Huang, B. 2013. Dynamic imaging of genomic loci in living human cells by an optimized CRISPR/Cas system. Cell 155: 1479-1491.

Chen, B., Guan, J. and Huang, B. 2016a. Imaging Specific Genomic DNA in Living Cells. Annu. Rev. Biophys. 45: 1-23.

Chen, B., Hu, J., Almeida, R., Liu, H., Balakrishnan, S., Covill-Cooke, C., Lim, W. A. and Huang, B. 2016b. Expanding the CRISPR imaging toolset with Staphylococcus aureus Cas9 for simultaneous imaging of multiple genomic loci. Nucleic Acids Res. 44: e75.

Ebadi-Almas, D., Karimzadeh, G. and Mirzaghaderi, G. 2012. Karyotypic variation and karyomorphology in Iranian endemic ecotypes of Plantago ovata Forsk. Cytologia 77: 215-223.

Fang, Y. and Spector, D. L. 2005. Centromere positioning and dynamics in living Arabidopsis plants. Mol. Biol. Cell 16: 5710-5718.

Feng, C. M., Qiu, Y., Van Buskirk, E. K., Yang, E. J. and Chen, M. 2014. Light-regulated gene repositioning in Arabidopsis. Nat. Commun. 5: 3027.

Fu, Y., Rocha, P. P., Luo, V. M., Raviram, R., Deng, Y., Mazzoni, E. O. and Skok, J. A. 2016. CRISPR-dCas9 and sgRNA scaffolds enable dual-colour live imaging of satellite sequences and repeat-enriched individual loci. Nat. Commun. 7: 11707.

Fujimoto, S., Sugano, S. S., Kuwata, K., Osakabe, K. and Matsunaga, S. 2016. Visualization of specific repetitive genomic sequences with fluorescent TALEs in Arabidopsis thaliana. J. Exp. Bot. 67: 6101-6110

Fujimoto, S., Yonemura, M., Matsunaga, S., Nakagawa, T., Uchiyama, S. and Fukui, K. 2005. Characterization and dynamic analysis of Arabidopsis condensin subunits, AtCAP-H and AtCAP-H2. Planta 222: 293-300.

Gaj, T., Gersbach, C. A. and Barbas, C. F. 3rd 2013. ZFN, TALEN, and CRISPR/Cas-based methods for genome engineering. Trends Biotechnol. 31: 397-405.

Gerlich, D., Beaudouin, J., Kalbfuss, B., Daigle, N., Eils, R. and Ellenberg, J. 2003. Global chromosome positions are transmitted through mitosis in mammalian cells. Cell 112: 751-764.

Higashi, T., Matsunaga, S., Isobe, K., Morimoto, A., Shimada, T., Kataoka, S., Watanabe, W., Uchiyama, S., Itoh, K. and Fukui, K. 2007. Histone H2A mobility is regulated by its tails and acetylation of core histone tails. Biochem. Biophys. Res. Commun. 357: $627-632$.

Hirakawa, T., Katagiri, Y., Ando, T. and Matsunaga, S. 2015. DNA double-strand breaks alter the spatial arrangement of homolo- gous loci in plant cells. Sci. Rep. 5: 11058.

Hirakawa, T. and Matsunaga, S. 2016a. Three-dimensional, live-cell imaging of chromatin dynamics in plant nuclei using chromatin tagging systems. Methods Mol. Biol. 1469: 189-195.

Hirakawa, T. and Matsunaga, S. 2016b. Chromatin tagging systems contribute to live imaging analyses for chromatin dynamics. Cytologia 81: 121-123.

Hizume, M., Shiraishi, H., Matsusaki, Y. and Shibata, F. 2013. Localization of $45 \mathrm{~S}$ and $5 \mathrm{~S}$ rDNA on chromosomes of Nigella damascena, Ranunculaceae. Cytologia 78: 379-381.

Hoshi, Y., Yagi, K., Matsuda, M., Matoba, H., Tagashira, N., Pląder, W., Malepszy, S., Nagano, K. and Morikawa, A. 2011. A comparative study of the three cucumber cultivars using fluorescent staining and fluorescence in situ hybridization. Cytologia $\mathbf{7 6}$ : 3-10.

Ikeda, K., Sato, S., Matoba, H., Nagano, K. and Uchiyama, H. 2013. Molecular cytogenetic analysis of the critically endangered Trigonotis radicans var. radicans and var. sericea and allied species in Japan. Cytologia 78: 297-303.

Jinek, M., Chylinski, K., Fonfara, I., Hauer, M., Doudna, J. A. and Charpentier, E. 2012. A programmable dual-RNA-guided DNA endonuclease in adaptive bacterial immunity. Science 337: 816-821.

Jovtchev, G., Borisova, B. E., Kuhlmann, M., Fuchs, J., Watanabe, K., Schubert, I. and Mette, M. F. 2011. Pairing of lac O tandem repeats in Arabidopsis thaliana nuclei requires the presence of hypermethylated, large arrays at two chromosomal positions, but does not depend on H3-lysine-9-dimethylation. Chromosoma 120: 609-619.

Kanda, T., Sullivan, K. F. and Wahl, G. M. 1998. Histone-GFP fusion protein enables sensitive analysis of chromosome dynamics in living mammalian cells. Curr. Biol. 8: 377-385.

Kantek, D. L. Z., Peres, W. A. M. and Moreira-Filho, O. 2015. Cytogenetics of Trichomycterus brasiliensis (Siluriformes: Trichomycteridae) from the upper São Francisco River basin (MG). Cytologia 80: 25-29.

Kimura, H. and Cook, P. R. 2001. Kinetics of core histones in living human cells: little exchange of $\mathrm{H} 3$ and $\mathrm{H} 4$ and some rapid exchange of H2B. J. Cell Biol. 153: 1341-1353.

Kuroki, Y., Shibata, F. and Hizume, M. 2013. Chromosome bandings and signal pattern of FISH using rDNAs in Bellevalia romana. Cytologia 78: 399-401.

Lane, A. B., Strzelecka, M., Ettinger, A., Grenfell, A. W., Wittmann, T. and Heald, R. 2015. Enzymatically generated CRISPR libraries for genome labeling and screening. Dev. Cell 34: 373-378.

Lermontova, I., Schubert, V., Fuchs, J., Klatte, S., Macas, J. and Schubert, I. 2006. Loading of Arabidopsis centromeric histone CENH3 occurs mainly during G2 and requires the presence of the histone fold domain. Plant Cell 18: 2443-2451.

Lindhout, B. I., Fransz, P., Tessadori, F., Meckel, T., Hooykaas, P. J. and van der Zaal, B. J. 2007. Live cell imaging of repetitive DNA sequences via GFP-tagged polydactyl zinc finger proteins. Nucleic Acids Res. 35: e107.

Lombello, R. A. and Pinto-Maglio, C. A. F. 2014. Cytogenetics and Reproductive Biology of Bixa orellana L. (Bixaceae). Cytologia 79: $379-386$.

Lottersberger, F., Karssemeijer, R. A., Dimitrova, N. and de Lange, T. 2015. 53BP1 and the LINC complex promote microtubule-dependent DSB mobility and DNA repair. Cell 163: 880-893.

Ma, H., Naseri, A., Reyes-Gutierrez, P., Wolfe, S. A., Zhang, S. and Pederson, T. 2015. Multicolor CRISPR labeling of chromosomal loci in human cells. Proc. Natl. Acad. Sci. U.S.A. 112: 3002-3007.

Ma, H., Reyes-Gutierrez, P. and Pederson, T. 2013. Visualization of repetitive DNA sequences in human chromosomes with transcription activator-like effectors. Proc. Natl. Acad. Sci. U.S.A. 
110: $21048-21053$.

Ma, H., Tu, L. C., Naseri, A., Huisman, M., Zhang, S., Grunwald, D. and Pederson, T. 2016. Multiplexed labeling of genomic loci with dCas9 and engineered sgRNAs using CRISPRainbow. Nat. Biotechnol. 34: 528-530.

Matsunaga, S. 2016. FISH is in the limelight again beyond a cytogenetical technique for metaphase chromosomes. Cytologia 81: $3-6$.

Matsunaga, S., Katagiri, Y., Nagashima, Y., Sugiyama, T., Hasegawa, J., Hayashi, K. and Sakamoto, T. 2013. New insights into the dynamics of plant cell nuclei and chromosomes. Int. Rev. Cell Mol. Biol. 305: 253-301.

Matsunaga, S. and Umeda, M. 2011. DNA Double-strand Breaks Induce Endoreduplication. Cytologia 76: 229-230.

Miyanari, Y. 2016. A new approach to dissect nuclear organization: TALE-mediated Genome Visualization (TGV). Methods Mol. Biol. 1338: 89-97.

Miyanari, Y., Ziegler-Birling, C. and Torres-Padilla, M. E. 2013. Live visualization of chromatin dynamics with fluorescent TALEs. Nat. Struct. Mol. Biol. 20: 1321-1324.

Moscou, M. J. and Bogdanove, A. J. 2009. A simple cipher governs DNA recognition by TAL effectors. Science 326: 1501.

Nozaki, T., Imai, R., Tamura, S. and Maeshima, K. 2014. Single nucleosome imaging in living human cells. Cytologia 79: 1-2.

Ochiai, H., Sugawara, T. and Yamamoto, T. 2015. Simultaneous live imaging of the transcription and nuclear position of specific genes. Nucleic Acids Res. 43: e127.

Pontvianne, F., Blevins, T., Chandrasekhara, C., Mozgová, I., Hassel, C., Pontes, O. M., Tucker, S., Mokros, P., Muchová, V., Fajkus, J. and Pikaard, C. S. 2013. Subnuclear partitioning of rRNA genes between the nucleolus and nucleoplasm reflects alternative epiallelic states. Genes Dev. 27: 1545-1550.

Robinett, C. C., Straight, A., Li, G., Willhelm, C., Sudlow, G., Murray, A. and Belmont, A. S. 1996. In vivo localization of DNA sequences and visualization of large-scale chromatin organization using lac operator/repressor recognition. J. Cell Biol. 135: $1685-1700$.

Rosa, S., De Lucia, F., Mylne, J. S., Zhu, D., Ohmido, N., Pendle, A., Kato, N., Shaw, P. and Dean, C. 2013. Physical clustering of FLC alleles during Polycomb-mediated epigenetic silencing in vernalization. Genes Dev. 27: 1845-1850.

Ruan, G. P., Wang, J. X., Yao, X., Pang, R. Q., Cai, X. M., He, J. and Pan, X. H. 2013. Different hematopoietic reconstruction abilities of transplanted cells from bone marrow, spleen, liver and peripheral blood. Cytologia 78: 113-119.

Schneider, R. and Grossched1, R. 2007. Dynamics and interplay of nuclear architecture, genome organization, and gene expression. Genes Dev. 21: 3027-3043.

Sexton, T., Schober, H., Fraser, P. and Gasser, S. M. 2007. Gene regulation through nuclear organization. Nat. Struct. Mol. Biol. 14:
1049-1055.

Shao, S., Zhang, W., Hu, H., Xue, B., Qin, J., Sun, C., Sun, Y., Wei, W. and Sun, Y. 2016. Long-term dual-color tracking of genomic loci by modified sgRNAs of the CRISPR/Cas9 system. Nucleic Acids Res. 44: e86.

Shibata, F. and Hizume, M. 2011. Survey of Arabidopsis- and human-type telomere repeats in plants using fluorescence in situ hybridisation. Cytologia 76: 353-360.

Shibata, F. and Hizume, M. 2015. Multi-color fluorescence in situ hybridization. Cytologia 80: 385-392.

Shirakawa, J., Nagano, K. and Hoshi, Y. 2012. Polyploid genome structure of Drosera spatulata complex (Droseraceae). Cytologia 77: 97-106.

Suto, Y., Akiyama, M., Gotoh, T. and Hirai, M. 2013. A modified protocol for accurate detection of cell fusion-mediated premature chromosome condensation in human peripheral blood lymphocytes. Cytologia 78: 97-103.

Suto, Y., Hirai, M., Akiyama, M., Suzuki, T. and Sugiura, N. 2012. Sensitive and rapid detection of centromeric alphoid DNA in human metaphase chromosomes by PNA fluorescence in situ hybridization and its application to biological radiation dosimetry. Cytologia 77: 261-267.

Takata, H., Matsunaga, S., Morimoto, A., Ono-Maniwa, R., Uchiyama, S. and Fukui, K. 2007. H1.X with different properties from other linker histones is required for mitotic progression. FEBS Lett. 581: $3783-3788$.

Tanenbaum, M. E., Gilbert, L. A., Qi, L. S., Weissman, J. S. and Vale, R. D. 2014. A protein-tagging system for signal amplification in gene expression and fluorescence imaging. Cell 159: 635-646.

Thanisch, K., Schneider, K., Morbitzer, R., Solovei, I., Lahaye, T., Bultmann, S. and Leonhardt, H. 2014. Targeting and tracing of specific DNA sequences with dTALEs in living cells. Nucleic Acids Res. 42: e38.

Wang, S., Su, J. H., Zhang, F. and Zhuang, X. 2016. An RNAaptamer-based two-color CRISPR labeling system. Sci. Rep. 6: 26857.

Yokomi, I., Ogiwara, H., Kohno, T., Yokota, J. and Satoh, H. 2013. Comparative fiber-FISH reveals what happened at the integration site of the transfected plasmid DNA. Cytologia 78: 121-122.

Yokoyama, R., Hirakawa, T., Hayashi, S., Sakamoto, T. and Matsunaga, S. 2016. Dynamics of plant DNA replication based on PCNA visualization. Sci. Rep. 6: 29657.

Yuan, K. and O'Farrell, P. H. 2016. TALE-light imaging reveals maternally guided, $\mathrm{H} 3 \mathrm{~K} 9 \mathrm{me} 2 / 3$-independent emergence of functional heterochromatin in Drosophila embryos. Genes Dev. 30: 579-593.

Yuan, K., Shermoen, A. W. and O'Farrell, P. H. 2014. Illuminating DNA replication during Drosophila development using TALElights. Curr. Biol. 24: R144-R145. 